INGURUAK [71] | $2021 \mid$ 1-6

http://dx.doi.org/10.18543/inguruak-71-2021-art01

ISSN 0214-7912

\title{
Presentación \\ Lo que la COVID-19 nos enseñó: ciencia, divulgación y futuro
}

What COVID-19 Taught Us: Science, Outreach, and the Future

\author{
Iraide Fernández Aragón* \\ Directora de Inguruak \\ Universidad del País Vasco/Euskal Herriko Unibertsitatea (UPV/EHU)
}

La crisis sanitaria originada por la irrupción de la COVID-19 en marzo de 2020 generó una situación sin precedentes a nivel internacional. Ni como sociedad, ni institucionalmente, estábamos preparadas para gestionar tamaño caos y, tras algunas respuestas rápidas, aunque ineficaces, comenzó la frenética carrera científica. Personas e instituciones se aferraron a la ciencia para hacer frente a la incertidumbre como antaño se aferraron a la religión. Un cambio cualitativo relevante, desde luego. Comenzó así la era dorada de la ciencia (Menéndez Velázquez, 2021), un tiempo en el que las decisiones políticas más transcendentales tienen su base en los resultados de las últimas investigaciones científicas y los avances científicos ocupan el prime time televisivo.

La crisis sanitaria ocultaba una crisis social y económica que, poco a poco, se fue revelando, junto con la necesidad de transcender el «nacionalismo metodológico» o las barreras del Estado-Nación para explicar una crisis que no pude circunscribirse geopolíticamente (Sassen, 2007). La ciencia se dispuso a reducir el espacio de la incertidumbre, las ciencias naturales elaborando vacunas y las ciencias sociales realizando diagnósticos y tratando de comprender la nueva realidad social y económica (Fernández Esquinas, 2020), pero, sobre todo, resaltando las desigualdades y situaciones de vulnerabilidad que la pandemia y su gestión estaban generando (Fernández Aragón et al., 2021). En este sentido, destaca la intrincada relación entre salud y clase social ante un virus que no afecta por igual a todas la personas o colectivos y que casi reproduce las relaciones de dominación social (Nuño de la Rosa, 2021). De forma semejante ocurre entre países, ya que el acceso a la vacunación y a los sistemas de salud se da de manera muy desigual entre los llamados

\footnotetext{
* Correspondencia a/Correspondence to: Iraide Fernández Aragón. Universidad del País Vasco/Euskal Herriko Unibertsitatea (UPV/EHU) - iraide.fernandez@ ehu.eus - https://orcid.org/0000-0003-0669-7155

Cómocitar/Howtocite: FernándezAragón,Iraide(2021).«LoquelaCOVID-19nosenseñó:ciencia,divulgaciónyfuturo»;/Inguruak,71,1-6.(http://dx.doi.org/10.18543/inguruak-71-2021-art01). Recibido/Received: 2 diciembre 2021; Versión final/Final version: 25 diciembre 2021.

ISSN 0214-7912 / @ 2021 UPV/EHU
}

(c) (i) Esta obra está bajo una Licencia

Creative Commons Atribución-NoComercial-SinDerivadas 4.0 Internacional 
PRESENTACIÓN. Lo que la COVID-19 nos enseñó: ciencia, divulgación y futuro | Iraide Fernández Aragón

países pobres y ricos (Sanahuja, 20201). Sin embargo, más allá de considerar el papel central de la investigación en este contexto y en el marco de la globalización, la reconfiguración del panorama mundial exige una reflexión en torno a las condiciones en las que la ciencia llega a esta pandemia y se desarrolla posteriormente.

No cabe duda de que la pandemia ha puesto de manifiesto la importancia de la ciencia para superar una crisis de estas características, cuestión similar a resaltar la necesidad de invertir en ciencia para obtener resultados. Efectivamente, uno de los grandes retos políticos del escenario pospandémico será financiar, orientar y utilizar las ciencias sociales de cara a la gestión de «la nueva normalidad» (Fernández Esquinas, 2020). Podría afirmarse que ha sido necesaria una crisis sanitaria internacional para colocar en el centro de la estrategia de recuperación a la ciencia, en concreto el I+D+I, tras años de recortes presupuestarios (Cotec, 2020). De hecho, en julio de 2020 el gobierno central presentaba el «Plan de choque por la Ciencia y la Innovación», que incluía 1.056 millones de euros de inversión directa (Gobierno de España, 2021). Y, sin embargo, este impulso necesario no soluciona los problemas y demandas de la investigación, que arrastra algunos problemas estructurales que deben solventarse (Codina, 2016).

Ubicar a la ciencia y la investigación en el plano que les corresponde es prioritario para alimentar su capacidad de respuesta ante un cambio acelerado o crisis (sanitaria, como esta; o económica como la de 2008). Es decir, la vacuna que da solución a la crisis o las repuestas que nos ayuden a gestionarla no surgen «de la nada». Es necesario que, previamente, exista una estructura física y organizacional (grupos de investigación), investigadores/as, financiación a los grupos, becas y espacios de divulgación para el conocimiento: entre otros, revistas científicas. Asimismo, cabe destacar que, si deseamos que los resultados obtenidos de ese avance científico sean públicos, pública debería ser también la financiación. La suma de todas estas cuestiones genera un caldo de cultivo en el que surge la ciencia, una estructura estable que puede generar de manera rápida y flexible las ansiadas respuestas cuando llegan las grandes preguntas. Sin embargo, la investigación no se encontraba en su mejor momento cuando irrumpió la COVID-19. Según los datos publicados por el Observatorio Español I+D+I (ICONO), España dedica tan solo el 1,2\% de su PIB al gasto en investigación, porcentaje que sitúa al Estado en los peores puestos europeos y muy por debajo de la media (2\%). En este sentido, no es de extrañar que, en 2019, el número de personas investigadoras por cada millón de habitantes en el Estado fuera 2.944, mientras que en Europa la media ascendía a 4.066 (UNESCO, 2019).

La situación de desamparo de la ciencia y la investigación proviene de la anterior crisis económica, la que comenzó en 2008 con la caída de Lehman Brothers (Cotec, 2020). En ese contexto, se consideró que la investigación no era un ámbito prioritario, llevándose a cabo

\footnotetext{
${ }^{1}$ Mientras en los países con bajos ingresos únicamente el 4\% de la población ha recibido al menos una dosis, los países considerados desarrollados han recibido la pauta completa y se disponen a ampliar a la tercera dosis (Mathew and Roser, 2021).
} 
PRESENTACIÓN. Lo que la COVID-19 nos enseñó: ciencia, divulgación y futuro | Iraide Fernández Aragón

un recorte presupuestario significativo: 9.673 millones de euros en 2009 que pasaron a ser 6.394 en 2012 (ICONO, 2020). Una década perdida para el avance científico, los grupos de investigación y las personas investigadoras, que tuvieron que interrumpir sus proyectos, carreras y biografías. Esas mismas personas y grupos a los que, con la llegada de la crisis sanitaria, se les exigió una respuesta rápida y contundente.

La precarización de la investigación en el ámbito universitario es una demanda de personas investigadoras y sindicatos desde hace años. Según los últimos datos, en la Universidad del País Vasco/Euskal Herriko Unibertsitatea existen 1.334 contratos de investigación, de los cuales el $48 \%$ son personal investigador predoctoral, es decir, los futuros investigadores/as de la sociedad, con salarios medios de 1.067€ (LAB, 2021). Un salario que no se corresponde con la relevancia y cualificación del puesto y que va acompañado de una elevada precariedad e inestabilidad. Debemos garantizar que las personas investigadoras realicen una carrera definida y estable o enfrentarnos al hecho de que estas personas busquen otros contextos que no les precaricen y les ofrezcan seguridad vital. Así lo manifiesta el Observatorio de Investigación e Innovación de la Comisión Europea, según el cual, España perdió 12.000 científicos/as entre 2010 y 2015 (Roberge y Campbell, 2021). Y, en este sentido, debemos garantizar también el papel de las mujeres en la ciencia, asumiendo y rompiendo el escandaloso techo de cristal que existe en el ámbito académico: según los datos del informe publicado por Ikerbasque las diferencias entre hombres y mujeres van aumentando a medida que avanza la carrera investigadora. Así, aunque el número de estudiantes de doctorado es similar, son menos las mujeres que continúan en la ciencia tras obtenerlo y, aún menos, las que consolidan posiciones estables o lideran sus propios grupos de investigación (IKERBASQUE, 2021). No se trata solo de la brecha STEM ${ }^{2}$, también de la necesidad de establecer medidas que favorezcan la corresponsabilidad en los cuidados y asegurar los derechos de conciliación de la vida laboral y familiar. Tal vez así, la brecha existente entre los artículos publicados por investigadores e investigadoras se acorte y aumente el número de investigadoras principales (Ministerio de Ciencia e Innovación, 2021). La sociedad deposita elevadas expectativas sobre las personas investigadoras, tanto es así que, como afirma Noam Chomsky, tienen la ingente tarea de rastrear la verdad entre el discurso oficial e investigar y conocer si existen motivos para ponerlo en duda (Chomsky, 2019). No obstante, esa responsabilidad debe ir acompañada de una valoración social y laboral que responda a las exigencias sociales e institucionales y que se de en términos de igualdad (especialmente de género).

En definitiva, si queremos una ciencia en buena forma y lista para los retos del futuro (que sin duda se presentarán) la investigación debe convertirse en un ámbito prioritario para sociedad e instituciones. A este respecto, uno de los grandes retos de esta pandemia, y de otras situaciones pasadas y futuras, es la comunicación. La primera pandemia mundial en la era de las redes sociales ha provocado numerosos desafíos en el ámbito de la comunicación, especialmente, en lo relacionado con las fake news (Catalán-Marotos, 2020). Si

\footnotetext{
${ }^{2}$ Menor presencia de mujeres en carreras científicas o tecnológicas (Shapiro y Williams, 2012).
} 
PRESENTACIÓN. Lo que la COVID-19 nos enseñó: ciencia, divulgación y futuro | Iraide Fernández Aragón

bien la carrera electoral y posterior presidencia de Trump ya generó temor sobre los riesgos de las fake news para el sistema democrático (Cabezuelo y Manfredi, 2019), la pandemia ha elevado esos temores a certezas. La rápida propagación de mensajes falsos, a menudo ligados a las posiciones negacionistas, en plataformas como Facebook, Instagram o Twitter hizo que la Organización Mundial de la Salud acuñara el término de «infomedia», francamente preocupada por la incidencia de estos mensajes en la salud pública (CatalánMarotos, 2020). Resulta prioritario examinar los determinantes que han provocado esta situación. La suma de la pérdida de legitimidad de las fuentes de autoridad científica global (Nuño de la Rosa, 2021); la falta de transparencia y divulgación de algunos resultados científicos (Menéndez Velázquez, 2021); y la aparición de nuevos generadores de opinión o influencers, cuyo contenido no se regula, dan como resultado una suerte de realidad donde la verdad no es una variable relevante para el proceso de información. La única solución para una situación en la que identificar el conocimiento científico y los mensajes falsos es complicado pasa por el fortalecimiento de la transferencia de conocimiento científico de la academia a la sociedad (López-Borrull, 2020).

En este sentido, se detecta la necesidad de realizar diversos cambios en la forma en la que la academia lleva a cabo su actividad. En primer lugar, es necesaria una mayor alfabetización científica dada la mayor presencia de contenidos científicos en el debate y opinión social (López-Borrull, 2020). En segundo lugar, el avance científico debe ser público y abierto, de acceso fácil para la ciudadanía y basado en el Open Science (Menéndez Velázquez, 2021). La clave para acelerar el progreso científico y democratizar la ciencia es la transmisión abierta del conocimiento generado y, en este horizonte más que deseable, las revistas científicas tienen un papel esencial (López y Cordero, 2005).

Los descubrimientos científicos no constituyen un avance real si no se dan a conocer sus resultados y conlusiones más relevantes con el resto de la comunidad científica que, a su vez, empleará ese conocimiento para mejorarlo y volver a difundirlo, generando así un intrincado sistema social con canales, normas y principios propios (Mendoza y Paravic, 2006). Las publicaciones científicas así entendidas, aunque surgieron hace más de dos siglos, siguen considerándose básicas para el proceso de transferencia científico $\mathrm{y}$, si garantizan su accesibilidad libre y abierta, se transforman en un archivo público del conocimiento. Los sesudos y costosos procesos de evaluación garantizan la veracidad y calidad de los resultados de investigación publicados, convirtiéndose así en esa fuente fiable de conocimiento que tanto demandamos en el contexto de posverdad. Esta es la responsabilidad y función que se atribuye a la labor editorial en la ciencia, así pues, su valoración y financiación debería acompañar a la magnitud de la tarea que le ocupa. Tal vez esta misma magnitud es la que, a veces, convierte a las revistas científicas en lugares hostiles para las propias personas investigadoras. Bajo la máxima del publish or perrish (publicar o morir) y bajo el peso de los criterios de las agencias evaluadoras de calidad, es sencillo perder la perspectiva editorial y pasar por alto el verdadero objetivo de las revistas científicas: que las personas investigadoras, de todo tipo y condición, puedan publicar sus avances y compartirlos con el resto de la comunidad científica y la sociedad bajo criterios de calidad y éticos. 
PRESENTACIÓN. Lo que la COVID-19 nos enseñó: ciencia, divulgación y futuro | Iraide Fernández Aragón

Esta es la base sobre la que el equipo editorial de INGURUAK comienza este nuevo periodo, en el convencimiento de que ser un canal de comunicación abierto e inclusivo es posible. Donde las personas investigadoras puedan publicar de manera gratuita, en euskera o castellano y abierto para quien desee leerlo. Mejorar la calidad de la publicación sin perder su carácter local. Sin perder el objetivo de compartir, difundir, reconocer y colaborar ya que, si esto es esencial para el común de las ciencias, aún lo es más para la hermana menos valorada: la ciencia social. Precisamente, esa que debería jugar un papel protagonista cuando se da una ruptura o cambio social, como los que están aconteciendo en esta crisis sanitaria-social-económica.

A modo de conclusión, la autora de este texto espera que esta crisis sirva, al menos, para aprender la importancia de cuidar la estructura científica y las personas que la alimentan. $\mathrm{Si}$, como sociedad, anhelamos una ciencia social que responda en momentos convulsos; que cumpla el papel de ofrecer certidumbres (Luna-Nemecio, 2020); que investigue para orientar las decisiones políticas (Fernández Esquinas, 2020); que resalte las desigualdades y vulnerabilidades del sistema global (Nuño de la Rosa, 2021); y que genere conocimiento científico y de calidad frente a las fake news (Catalán-Matamoros, 2020), tendrá que priorizarse, valorarse y, sobre todo, financiarse.

\section{BIBLIOGRAFÍA}

Cabezuelo Lorenzo, F. y Manfredi, J. L. (2019). Posverdad, fake-news y agenda política en el discurso de Trump en Twitter. Historia y comunicación social , 24 (2), 449-483.

Catalán-Matamoros, D. (2020). La comunicación sobre la pandemia del COVID-19 en la era digital: manipulación informativa, fake news y redes sociales. Revista Española de Comunicación en Salud, 1, 5-8. https://doi.org/10.20318/recs.2020.5531

Chomsky, N. (2020). La responsabilidad de los intelectuales. Madrid: Sexto piso.

Codina, L. (2016). Evaluación de la ciencia: tan necesaria como problemática. El profesional de la información, v. 25, n. 5, pp. 715-719. https://doi.org/10.3145/epi.2016.sep.01

COTEC (2020). Informe Cotec 2020. Recuperado de: https://cotec.es/en/observacion/informecotec-2020/38c863cc-124a-4b04-a13f-059d19ddf47b

Mathieu, E. y Roser, M. (2021). How do death rates from COVID-19 differ between people who are vaccinated and those who are not? Our Wordl in data. Recuperado de: https://ourworldindata.org/covid-deaths-by-vaccination

Fernández Aragón, I. et al. (2021). Análisis de la desigualdad urbana. Propuesta de un Índice Sintético de Vulnerabilidad Urbana Integral (ISVUI) en Bilbao. ACE: Architecture, City and Environment, 15(45), 9520. http://dx.doi.org/10.5821/ace.15.45.9520 
PRESENTACIÓN. Lo que la COVID-19 nos enseñó: ciencia, divulgación y futuro | Iraide Fernández Aragón

Fernández Esquinas, M. (2020). Sociología y Ciencias Sociales en tiempos de crisis pandémica. Revista de Sociología de la Educación-RASE, 13 (2)Especial, COVID-19, 105-113. http://dx.doi.org/10.7203/RASE.13.2.17113

Gobierno de España (2021). Plan de choque para la ciencia y la innovación. Recuperado de: https://www.ciencia.gob.es/gesdamdoc-servlet/?uuid=23afa491-1b16-4e88-a90f-acce b3550355\&workspace $=$ dam\&formato $=$ pdf

Guillaume R. y David C. (2021). Provision and analysis of key indicators in research and innovation. Policy brief F - Scientific mobility. Luxembourg: Publications Office of the European Union. doi:10.2777/073061

ICONO (2020). Indicadores del sistema español de Ciencia, Tecnología e Innovación. Recuperado de: https://icono.fecyt.es/sites/default/files/filepublicaciones/indicadores_2020_web.pdf

IKERBASQUE (2021). Informe sobre la ciencia en Euskadi 2021. Recuperado de: https:// Www.ikerbasque.net/sites/default/files/files/INFORME\%20DE\%20CIENCIA\%20EUSKADI\%202021\%20II.pdf

LAB (2021). Informe personal docente e investigador de la UPV/EHU.

López, O. M. y Cordero, A. (2005). Un intento por definir las características generales de las revistas académicas electrónicas. Razón y Palabra, 43, 1-31.

Luna-Nemecio, J.M. (2020). Ciencias sociales y COVID-19: retos, vicisitudes y oportunidades para la investigación. FORHUM International Journal of Social Sciences and Humanities, 2(3), 6-12. https://doi.org/10.35766/jf20231

Mendoza, S. y Paravic, T. (2006). Origen, clasificación y desafíos de las Revistas Científicas. Investigación y Postgrado, 25, 1-20.

Menéndez Velázquez, A. (2021). Hacia una ciencia abierta, interdisciplinar y que conecte los mundos académico e industrial. Telos, 115.

Nuño de la Rosa, L. (2021). Ciencia y capitalismo en tiempos de covid. Viento sur, 178, 42-51.

Sassen, S. (2007) Una sociología de la globalización. Analisis político, 61, 3-27.

Shapiro, J. y Williams, A. (2012). The role of stereotype threats in undermining girls' and women's performance and interest in STEM fields. Sex Roles, 66, 175-183.

Singer, D. (2020). Pandemia y mundos posibles. BORDES, (16), 145-154.

UNESCO (2019). Number of Researchers per million inhabitants by country, United Nations Educational, Scientific and Cultural Organisation (UNESCO) Institute for Statistics. Recuperado de: http://uis.unesco.org/?URL_ID=3755\&URL_DO=DO_TOPIC\&URL_SECTION=201 